\title{
Automatic Composition of Instructional Units in Virtual Learning Environments
}

\author{
https://doi.org/10.3991/ijet.v13i06.8107 \\ Meriem Hnida ${ }^{\bowtie}$, Mohammed Khalidi Idrissi, Samir Bennani \\ Mohammed V University in Rabat, Morocco \\ meriemhnida@research.emi.ac.ma
}

\begin{abstract}
In this paper, a new approach for automatic composition of instructional units based on a new variant of Harmony Search Algorithm is proposed. The purpose is to solve curriculum sequencing issue by designing and arranging learning content in a suitable sequence. By suitable sequence we mean a learning sequence that fits learner level and presents the content in a way that conveys its structure to learner. Results show that the proposed approach is promising. For instance, individualized courseware plan are generated "on the spot" carefully considering both students characteristics and subjectmatter coherence.
\end{abstract}

Keywords-Curriculum Sequencing Problem, Curriculum Design, CS Problem, Virtual Learning Environment, Evolutionary Computation, Harmony Search Algorithm, Learning Path, e-Learning, Personalized Learning, Adaptive Teaching Learning Sequence.

\section{Introduction}

Technology changed drastically the teaching learning process. Using technology students benefit from instant access to materials anytime and anywhere. Online learning materials have the potential to captivate student's attention and foster their interest since this generation is digital natives. Many studies pointed out the importance of using technology in the educational settings. For instance, the survey conducted by[1] shows that students are highly motivated to adopt mobile technologies for educational purposes and that they consider mobile learning as a useful and easy to use learning method. Although technology provides a huge boost in flexibility. Still, most virtual learning environments provide students with "One-size-fits-all": the same learning material is presented in the same way and at the same time to all learners. This ignores the fact that learners have different backgrounds, knowledge and goals, they proceed according to their own pace and might be interested in different contents at different stages of the learning process. Another drawback of the "One-size-fits-all" is that, in an online educational environment, learner may get confused when searching for what could fit their level or learning style, leading up to online courses with not balanced learning concepts, or sometimes to cognitive overload. 
One of the current challenges of today's educational technology is to provide students with authentic and meaningful learning experience, helping them achieve a standard of performance, and succeed - at their own pace- in a highly competitive environment. However, information overload remains an issue in virtual learning environments where a massive amount of information is presented to learners in a way that not conveys to their level or learning style making them feel frustrated and dissatisfied.

To this end, this paper highlights a new approach for automatic composition of instructional units. More specifically, the focus is on the mechanism of generation of courseware taking into consideration both students characteristics and instructional design point of view. This is a well-known research issue that has been classified by many researchers as an NP hard problem. This has been justified by [2], in order to find an appropriate learning sequence for a subject-matter with $\mathrm{N}$ learning concepts, one should explore a large number of possible solutions with a Total of N!. In this case, using an ordinary algorithm to search for suitable learning concepts would take a very long time, and may end up without finding optimal learning sequence. It's important to note that: not only exploring large databases to form suitable learning sequences is time consuming and difficult task, it is also heavily constrained. Some of constraints are related to students' characteristics and others are bound up with domain knowledge.

The reminder of this article is organized as follows: in section 2, related works are reported and discussed. This section is divided into two main parts: first of all, approaches for curriculum design are presented and recent approaches for composition of instructional units are highlighted. In section 3, Automatic Composition of Instructional Units is presented. For instance, we present the proposed approach to solve the problem based on Harmony Search Algorithm followed by experiments and results in section 4. Section 5, summarizes the contribution. Finally, we draw a conclusion and announce our future work.

\section{Related works}

Creating individualized teaching learning sequence is a research issue commonly known as "Curriculum Sequencing Problem" or "Learning Path Generation" or "Personalized Teaching Learning Sequence". Curriculum is the arrangement of content. It compasses learning content in respect to an educational approach. It should be flexible and responsive to meet students' needs and expectations. Curriculum sequencing problem might be divided into two open problems: (1) curriculum design and (2) curriculum sequencing. Curriculum design entails the process of designing structuring and chunking the content, while curriculum sequencing is the mechanism of gathering learning concepts together such as contents, activities, and exercises to form a unique learning sequence. Curriculum design and curriculum sequencing are critical steps to deliver appropriate learning sequence. In this section, we report and discuss related works. 


\subsection{Approaches for Instructional Design}

As stated by [3] "Curriculum is not a monolithic construct", it entails the design of learning materials or reuse of existing ones in respect to an educational approach and should be aligned to some standards. Curriculum design approaches could be classified into two main categories: learner-centered curriculum sequencing and conceptrelated curriculum sequencing.

In one hand, learner-centered curriculum approach puts the emphasis on students' characteristics and their changing states of mastery. It is based on the underlying assumption that learners are in the heart of any educational system and curriculum should be derived from students' characteristics. It replaces traditional way lecturers use to deliver knowledge with a self-directed learning in which student is responsible of learning and is engaged in the process. At this stage, educational technology should be able to pinpoint student's characteristics and knowledge level. It should also help them grow in their understanding moving them from novice to expert status. Many works fall in this category such as [4]who proposed a style-based ant colony system to provide student with suitable learning path in respect to their learning style. Learning style stands for preferred ways to learn (visual, aural, reading/writing and kinesthetic). Chih-Ming Chen[5] postulates that learner ability is an important factor that should be taken into consideration while designing e-learning course. Authors distinguished between learning style and cognitive style. The learning style refers to underlying traits that leads to a learning behavior while cognitive style stands for preferred ways of learning. The same authors propose an adaptive system based on dimensions of Felder-Silverman's learning style. Other researchers put the focus on learner's level, prior knowledge or competency into the design of curriculum.

In the other hand, concept-related curriculum depends on domain knowledge. The focus is on knowledge structure and design instruction to achieve a learning goal. As posited by[6] most approaches focus on the content to be delivered and pedagogical approaches are not incorporated. To deal with this issue, some researchers deal with the depth and breadth of the content to be taught while others are interested in techniques to structure domain knowledge. For instance many techniques are proposed to deal with the instructional design step: graph based presentation of content such as ontology[7] or Bayesian network structure[8], Visual Educational Modeling [9], Using SCORM(Shareable Content Object Reference Model) provided with most learning management systems to create and enable exchange of content[10].

Brusilovsky[11] distinguishes between (1) Active Sequencing and (2) Passive Sequencing. In one hand, Active sequencing is the mechanism of preparing a learning sequence "on the spot" based on learner profile. It's goal-driven and cover the whole subject-matter. The purpose of active sequencing is to build the most suitable individual path to achieve a standard of performance based on the difference between student's current level of knowledge and the target one. For instance, active sequencing uses intelligent techniques in order to search for the best learning objects in a hyperspace. This assumes that the subject-matter is divided into a series of learning objects which are stored in different learning repositories and might be linked so to form a unique individually planned sequence of resources. In the other hand, Passive sequencing - called also remediation or remedial sequencing - addresses the learning 
difficulties by providing students with relevant items to correct the misunderstandings or overcome the situations that hinder their achievement.

Another popular approach for instructional planning is collaborative filtering used in recommender systems; the purpose is to find appropriate content that suits for a target user. These approaches start with clustering students into homogenous groups called also virtual communities of interests then provide each group with appropriate learning material.

\subsection{Approaches for Composition of Instructional Units}

Composition of Instructional Unit stands for the operation of populating a sequence with suitable learning concepts. There have been several approaches for composition of instructional units proposed by researchers. For instance, some authors formulate the automatic composition of instructional unit as a Constraint Satisfaction Problem[12]while other researchers formulate the problem as a Multi-Objective Optimization Problem[13] or Permutation problem[14][15].

Many techniques are proposed in order to solve the issue of automatic composition of Instructional Units: evolutionary computation such as GA (Genetic Algorithm)[2][16][17], ACO(Ant Colony Optimization)[18], PSO (Particle Swarm Optimization)[2], Artificial Neural Network(ANN)[19], Bayesian Network(BN)[8], Fuzzy Logic(FL)[20], Case-based Reasoning[21], Semantic Web[22], IRT(Item Response Theory)[23][24] are used. As result, evolutionary computation is increasingly popular in recent years and the literature is divided into two popular categories: Genetic Algorithm, and Particle Swarm Optimization. Both algorithms are applied to solve diverse types of problems related to the e-learning field. However, none of the scientific papers found so far has tested the potential of Harmony Search Algorithm to solve Curriculum Sequencing issue.

The motivation of choosing HSA is as follows:

- GA, PSO and HSA belong to non-deterministic class of algorithms so the solution may vary for each run. The quality of the obtained solution depends on the initial population, and the chosen parameters and operations.

- GA and PSO require a set of configurations. Furthermore, GA needs Chromosome encoding which is different from one problem to another. In the other hand, HSA could directly be implemented, all it needs is setting parameter such as objective function, stopping criterion.

- HSA is based on the principle that each new solution is better than all existing ones stored in a memory called Harmony Memory. Iteration after iteration, the algorithm starts to use candidate solution belonging to known high-quality solutions. HS algorithm generates a new vector after considering all of the existing vectors.

- Finally, HSA returns more than one good solution. It returns $\mathrm{N}$ best solutions. $\mathrm{N}$ refers to Harmony Memory Size. 


\section{Automatic Composition of Instructional Units}

In what follow Harmony Search Algorithm is presented followed by its application to produce automatic instructional units.

\subsection{The proposed Approach}

We propose to use Harmony Search Algorithm (HSA) to tackle curriculum sequencing issue. HSA is a population-based metaheuristic algorithm which was first introduced by Zong Woo Geem[25] and has been applied to solve the optimization problem of water distribution networks. As pointed out by [26][27], HSA has gained popularity due to the fast convergence speed. It's an algorithm which attempts to imitate jazz musicians especially those who play music together for the first time. For instance, they use improvisation and notes variations to find good harmony. Each musician in the group should find iteratively and over time the next note; to do so s/he refers to previously played notes and the context of music they are playing. Harmony search Algorithm uses this metaphor to iteratively produce a solution vector.

Our contribution: the process of searching for better harmonies in HSA is replaced by the process of searching for better learning concepts to form an individualized learning sequence, carefully considering both student's needs and content coherence. In the following section, HSA algorithm will be presented and discussed then, its application to automatic composition of instructional units will be illustrated.

\subsection{Harmony Search Algorithm}

HSA is a novel population-based meta-heuristic algorithm. The basic idea is adjusting the input (pitches) in order to obtain a perfect output (harmony)[28]. For instance, when musicians try to come up with a new harmony, they usually try previous combinations of pitches they remember. This is analogous to the process of finding an optimal learning path by combining different learning objects already stored in different learning repositories. HSA searches for a possible combination of candidate solutions, called also decision variables, the purpose is to minimize or maximize a fitness function, under a set of constraints. Figure 1 depicts the HSA Global Search Strategy.

\begin{tabular}{|l|}
\hline HSA Global Strategy \\
\hline Initialize the algorithm and problem parameters \\
While (Stopping Criteria is not met) \\
Improvise a new solution vector \\
If (New solution is better than the worst ever stored) \\
Update Memory with the new solution \\
Endif
\end{tabular}

Fig. 1. HAS Global Strategy

The harmony search algorithm works as following: 
Step1: Initialize the optimization problem and algorithm Parameters. HSA Algorithm uses a set of abbreviations described below:

- $\boldsymbol{X}_{\boldsymbol{i}}$ : decision variable.

- $N$ : Number of decision variables.

- HM: Harmony Memory, a memory system in form of a matrix; it contains the best solution vectors found within the search process.

- HMS: The number of solution vectors in HM.

- HMCR: Harmony Memory Consideration Rate explained in rule (1).

- PAR: Pitch Adjusting Rate, explained in rule (3).

- Ni: Number of iterations, it's the basis for terminating the optimization process.

The output of the initialization step is a HM Matrix with random decision variables. Each solution vector of the Harmony Memory is judged based on a cost function $f(x)$ that the algorithm tries to recursively optimize. Obtained value of $f(x)$ is stored in the last column of the Matrix.

$$
\mathbf{H M}=\left[\begin{array}{cccc|c}
x_{1}^{1} & x_{2}^{1} & \cdots & x_{n}^{1} & f\left(\mathbf{x}^{1}\right) \\
x_{1}^{2} & x_{2}^{2} & \cdots & x_{n}^{2} & f\left(\mathbf{x}^{2}\right) \\
\vdots & \cdots & \cdots & \cdots & \vdots \\
x_{1}^{H M S} & x_{2}^{H M S} & \cdots & x_{n}^{H M S} & f\left(\mathbf{x}^{H M S}\right)
\end{array}\right]
$$

The improvisation step: Generate a new possible solution. A new solution vector $\mathrm{X}^{\prime}=\left(\mathrm{X}_{1}{ }_{1}, \mathrm{X}_{2}, \mathrm{X}_{3}, \mathrm{X}_{4}^{\prime}, . . \mathrm{X}_{\mathrm{N}}\right)$ is generated based on three rules HMCR, Random Selection and PAR explained bellow, which decide on the value that will be assigned to each decision variable in the new solution vector. The generation of new solution vector is called improvisation.

Rules of selecting new candidate solutions are:

Rule 1: select candidate solution based on HMCR (Harmony Memory Consideration Rate): it refers to the probability that a specific candidate solution would come from existing Matrix HM, since it contains the best solutions found so far. The value of HMCR $(0<=\mathrm{HMCR}<=1)$ but usually greater than 0.5 .

Rule 2: select a candidate solution based on Random selection: it refers to the process of random Selection of a solution candidate with a probability of ( 1 - HMCR).

$$
x_{i}^{\prime} \leftarrow \begin{cases}x_{i}^{\prime} \in\left\{x_{i}^{1}, x_{i}^{2}, \ldots, x_{i}^{\mathrm{HMS}}\right\} & \text { with probability HMCR } \\ x_{i}^{\prime} \in X_{i} & \text { with probability (1- HMCR). }\end{cases}
$$

Rule 3: select candidate solution based on PAR (Pitch Adjustment Rate) the probability that a specific candidate solution in the new vector would be replaced by a new one assigned randomly within the bounds of the problem. 
Harmony memory update. Once the new solution vector (Harmony) is obtained using the above rules (1) (2) and (3). The goodness of the new generated solution is calculated. The goodness of a solution vector function is calculated using a function $f(x)$ that recursively calculate the cost of solution vector, each of which is a possible learning sequence. If the new solution is better than the worst solution stored in the $\mathrm{HM}$, then the worst solution so far is excluded and replaced with the new one with less violated constraints.

$\mathbf{H M}=\left[\begin{array}{cccc|c}x_{1}^{1} & x_{2}^{1} & \ldots & x_{n}^{1} & f\left(\mathbf{x}^{1}\right) \\ x_{1}^{2} & x_{2}^{2} & \ldots & x_{n}^{2} & f\left(\mathbf{x}^{2}\right) \\ \vdots & \ldots & \ldots & \ldots & \vdots \\ \hline x_{1}^{H M S} & x_{2}^{H M S} & & x_{n}^{H M S} & f\left(\mathbf{x}^{\text {HMS }}\right) \\ \hline\end{array}\right.$

Step1: Retrieves the worst solution vector stored in $\mathrm{HM}$

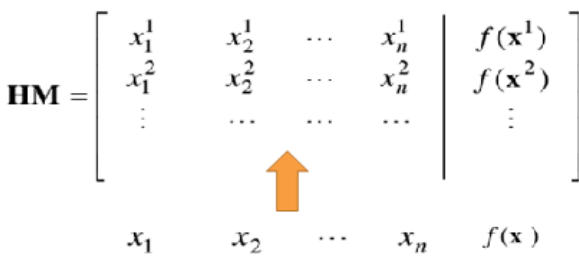

Step2: Includes the new solution vector in HM

Stopping Criterion. The computation is carried out until the algorithm meets the following stopping criterion:

- Maximum number of improvisations.

- Maximum number of iterations.

- Reaching a solution which satisfies all constraints.

\subsection{Harmony Search Algorithm For Automatic Composition of Instructional Units}

First of all, we explain the terminology related to the proposed variant of HSA in the following table, then we describe the algorithm steps.

Table 1.

\begin{tabular}{|c|l|}
\hline Variable & \multicolumn{1}{|c|}{ HSA Curriculum Sequence Terminology } \\
\hline $\mathrm{X} \mathrm{i}$ & Learning concept \\
\hline $\mathrm{N}$ & Number of concepts of a learning sequence \\
\hline $\mathrm{HM}$ & A set of learning sequence, each one is a vector of a Matrix \\
\hline $\mathrm{HMS}$ & The number of possible learning sequences that we bill generated by the algorithm. \\
\hline $\mathrm{HMCR}$ & $\begin{array}{l}\text { Harmony Memory Consideration Rate: The probability of choosing a learning concept that } \\
\text { will be assigned to a decision variable Xi from existing memory. }\end{array}$ \\
\hline $\mathrm{PAR}$ & $\begin{array}{l}\text { Pitch Adjustment Rate: the probability that a specific learning concept in the new learning } \\
\text { sequence would be replaced by a new one from a learning repository. }\end{array}$ \\
\hline $\mathrm{Ni}$ & Number of iterations, it's the basis for terminating the generation of instruction units. \\
\hline$\omega \mathrm{i}$ & Weight assigned by tutor to learning concept i. \\
\hline
\end{tabular}


In order to automatically generates individualized learning sequence, we set rules that will be used by the algorithm to select suitable learning concepts. This means that the arrangement of learning concepts should be drawn upon learner characteristics but is should also carefully consider intended learning outcomes and learning strategies. The algorithm should return an instructional plan in respect to the following constraints:

Constraint 1: difficulty parameter. Content should be organized in an order of increasing complexity; beginning with simple case and avoiding too complex ones, or concepts already mastered. It is also worth mentioning that learning concepts should be presented one a time with a respect of total amount of time.

Constraint2: Subject-matter coherence. Concepts have relationships to each other's and learning one thing may require student to learning something else first, or learning one concept should be complemented by another one. Relationships within learning concepts are calculated based on students' results on a pre-test. The result is a concept correlation matrix RC. A higher correlation means mutual relation of two or more concepts.

Figure 2 depicts the links between four concepts.

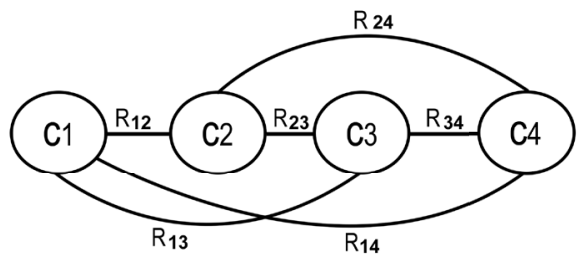

Fig. 2.

In order to determine how a concept is linked to other ones we used formula below:

$$
R C(i)=\sum_{j=1}^{N} R i j
$$

Each line of the matrix is a solution Vector $\left(X=\left\{X_{1}, X_{2}, X_{3}, X_{4} \ldots X_{N}\right)\right.$ is an ordered list of learning concepts, each learning concept is covered by a Learning Object. Each solution vector of the Harmony Memory is judged based on a cost function $\mathrm{f}(\mathrm{x})$ that the algorithm tries to recursively optimize. Obtained value of $f(x)$ is stored in the last column of the Matrix.

The purpose $f(x)$, in this case is to maximize $f(x)$ subject to $x i \in X i, i=1,2,3, \ldots, N$, with

$$
f(x)=\sum_{i=1}^{N} R C(i) * \omega i U i
$$


For a concept $\mathrm{i}$, Ui is the student level calculated based on a pretest, $\omega \mathrm{i}$ is the weight assigned by tutor, and RC(i) is the total correlation with other concepts.

HM is first filled with decision variables (Learning Concepts) generated randomly from a range of their possible values in the search space (Learning Repositories). One optional step is to rearrange, in the initialization step, the order of solution vectors according to $f(x)$, from best to worst solution. The following flow chart depicts the steps the proposed variant of HSA Algorithm.

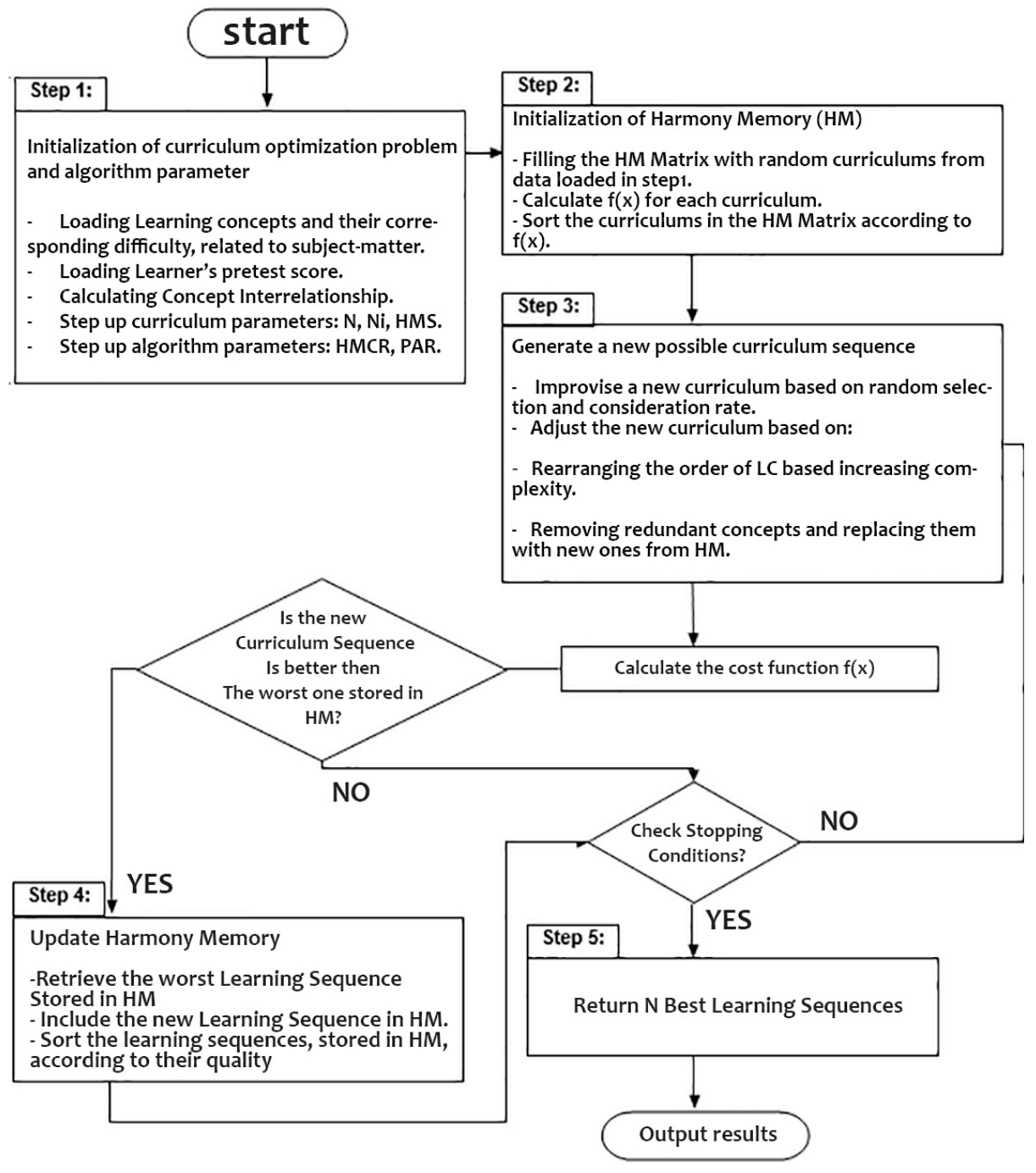

Fig. 3. Flow chart of the proposed variant of HSA algorithm 


\section{$4 \quad$ Experiments and results}

The purpose of the experiment is to evaluate the efficiency of HSA computational technique in finding the best instructional Units. In order to apply HSA for automatic composition of instructional units, both problem and algorithm parameters are specified. We run a simulation of a "database" courseware with 14 Learning Concepts with an increasing complexity. In order to calculate the relationships within concepts, we used recorded data from a pretest. Table 2 describes data used for experiments purpose.

Table 2. sample data used for experiments

\begin{tabular}{|c|l|}
\hline Id & \multicolumn{1}{|c|}{ Name } \\
\hline C1 & Relational databases \\
\hline C2 & Data Model \\
\hline C3 & Logical Model \\
\hline C4 & Normalization \\
\hline C5 & Create Table \\
\hline C6 & Alter Table \\
\hline C7 & Insert Query Syntax \\
\hline C8 & Select Query Syntax \\
\hline C9 & Multi-table queries \\
\hline C10 & Group By usage \\
\hline C11 & Aggregate functions \\
\hline C12 & Update Query Syntax \\
\hline C13 & Delete Query Syntax \\
\hline C14 & Using Restrictions \\
\hline
\end{tabular}

The experiment of the proposed HSA variant is conducted under eclipse IDE, the algorithm is implemented using java programing language, in a computer with JDK1.8, an Intel Core i5 and 4 GB RAM with Windows 10.0 operating system.

The experimental setup determines the performance of the proposed variant of Harmony Search Algorithm. For instance, speed and quality of the best learning sequence generated are important factors. In order to determine the computational cost, HSA is performed for 4500 iterations which stands for the number of evaluation of the fitness function, also considered as a basis for terminating the optimization process. Results

It has been observed that when multiplying the number of learning concepts, the algorithm speed remained reasonable. This important since the automatic composition of the instructional unit should be done "on the spot" right after the learner finishes the online assessment of perquisites. Figure 4 depicts HSA computational cost for generating instructional units. 


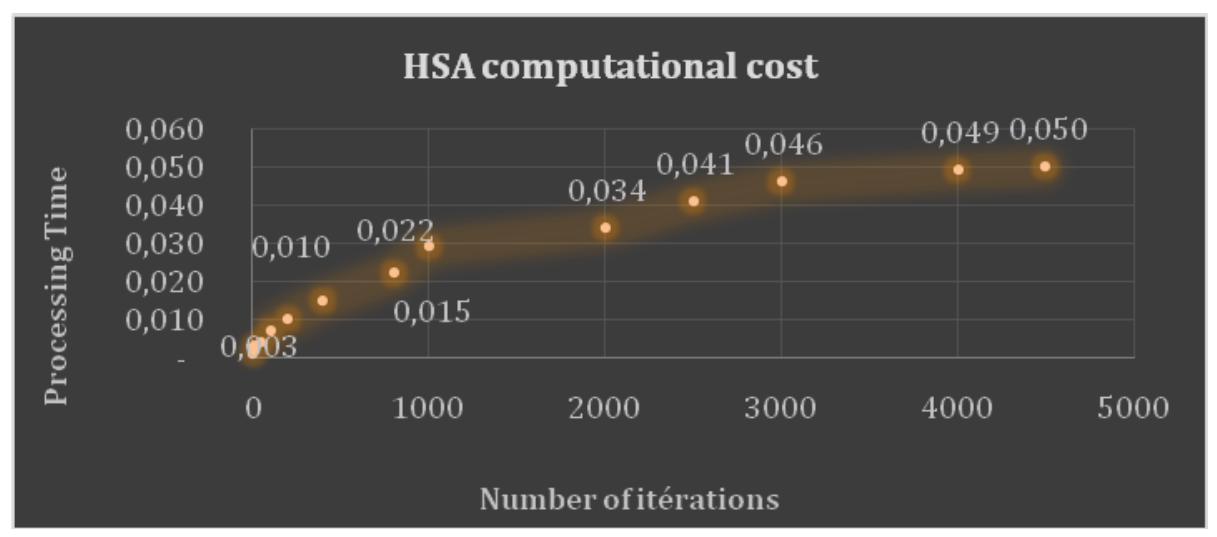

Fig. 4. HSA computational cost

Still, as in contrast with other algorithm the number of iterations, parameters and constraints influence the algorithm behavior. For instance, HMCR parameter influences the convergence of the algorithm. Lower HMCR parameter (between 0 and 0.5) leads the algorithm to converge quickly to local solution and higher HMCR parameter (between 0.5 and 1) leads the algorithm to optimal and global solution but may need a considerable number of iterations. The best solution is yet to find the finest HMCR values suitable to: number of learning concepts to search for and the number of learning concepts in learning repositories respectively.

We concluded that the proposed approach is promising. Pitch Adjustment Rate of Harmony Search Algorithm is modified so to fix three encountered issues: (1) Rearranging the order of Learning Concepts based on increasing complexity. (2) Removing redundant concepts and replacing them with new ones. (3) Taking into consideration that a learning sequence could entail less than $\mathrm{Ni}$ concepts for students who showed mastery for some Learning Concepts. The time window violation variable is not yet considered.

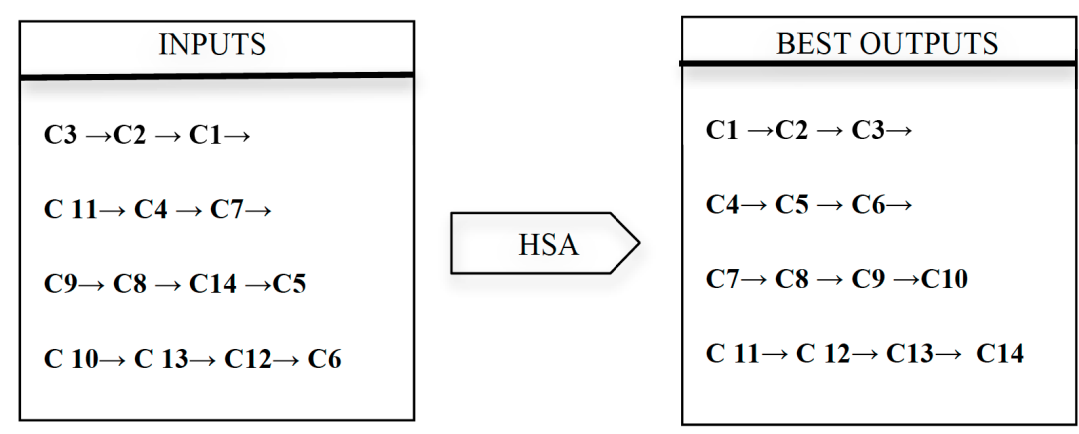

\begin{tabular}{|lllll|}
\hline With parameters & HMS: 5 & HMCR: 0.9 & PAR:0.6 & Ni : 4500 \\
\hline
\end{tabular}

Fig. 5. 


\section{Summary}

In this contribution, a new method to generate the learning path at beginning of online session is proposed. The purpose is to provide each learner with an individualized teaching learning sequence in a step by step process to achieve a standard of performance. More specifically, it is about selecting suitable learning concepts and sequencing them in a way that will be appropriate to student level of mastery with a respect to subject-matter structure.

The first constraint aims to provide students with content which fits their level and in an order of increasing complexity. The second constraint aims to ensure content coherence by maximizing relationships within concepts of the learning sequence. These relationships are calculated based on students' answers to a pretest. The result is a concept correlation matrix. The proposed algorithm uses the matrix to find a learning sequence which maximizes relationships within its concepts and presents learning concepts an order of increasing complexity, beginning with simple case and avoiding content that is too complex for student.

Each iteration, the algorithm improvises a new possible learning path, and then evaluates its goodness using a cost function. If the new learning path is better than the worst solution in the matrix, then the algorithm applies a harmony memory update to include the new solution vector.

As result, the Matrix (HM) contains $\mathrm{N}$ best solutions sorted according to their goodness. The output of the algorithm is N Best learning sequences. The best one yet is the first one on top of the matrix.

One advantage of HSA is its stability. For instance, when multiplying the number of learning concepts in the learning sequence and the number of learning objects in the search space, the algorithm speed remained reasonable

\section{Conclusion}

For several years great effort has been devoted to the study of the field of adaptive e-learning systems. Many researchers pointed out the importance of taking into consideration the learning characteristics in the design of those systems. We addressed the issue of automatic generation of instructional units with two sources of constraints: learner's current knowledge and interrelationship within a subject-matter. We proposed an intelligent mechanism of selecting and rearranging concepts into a learning sequence, using an adapted Harmony Search Algorithm. For future work, we intend to integrate the proposed algorithm into Moodle Learning Management System in order to generate instructional plan for bunch of learners and analyze some algorithm parameters. For instance, we would like to measure the extent to which generated curriculums fit each case of learners, this is by considering both students and teachers point of view. 


\section{$7 \quad$ References}

[1] O. F. Marzouki, M. K. Idrissi, and S. Bennani, "Effects of Social Constructivist Mobile Learning Environments on Knowledge Acquisition: A Meta-Analysis," Int. J. Interact. Mob. Technol., vol. 11, no. 1, pp. 18-39, Jan. 2017. https://doi.org/10.3991/ijim.v11i1. 5982

[2] L. de-Marcos, R. Barchino, J.-J. Martinez, J.-A. Gutierrez, and J.-R. Hilera, "CompetencyBased Intelligent Curriculum Sequencing: Comparing Two Evolutionary Approaches," in IEEE/WIC/ACM International Conference on Web Intelligence and Intelligent Agent Technology, 2008. WI-IAT '08, 2008, vol. 3, pp. 339-342. https://doi.org/10.1109/ WIIAT.2008.279

[3] S. Arafeh, "Curriculum mapping in higher education: a case study and proposed content scope and sequence mapping tool," J. Furth. High. Educ., vol. 40, no. 5, pp. 585-611, Sep. 2016. https://doi.org/10.1080/0309877X.2014.1000278

[4] T. I. Wang, K. Te Wang, and Y. M. Huang, "Using a style-based ant colony system for adaptive learning," Expert Syst. Appl., vol. 34, no. 4, pp. 2449-2464, May 2008. https://doi.org/10.1016/j.eswa.2007.04.014

[5] C. M. Chen, H. M. Lee, and Y. H. Chen, "Personalized e-learning system using Item Response Theory," Comput. Educ., vol. 44, no. 3, pp. 237-255, Apr. 2005. https://doi.org/10.1016/j.compedu.2004.01.006

[6] F. Yang, F. W. B. Li, and R. W. H. Lau, "A Fine-Grained Outcome-Based Learning Path Model,” IEEE Trans. Syst. Man, Cybern. Syst., vol. 44, no. 2, pp. 235-245, Feb. 2014. https://doi.org/10.1109/TSMCC.2013.2263133

[7] T. Lendyuk, A. Melnyk, S. Rippa, I. Golyash, and S. Shandruk, "Individual learning path building on knowledge-based approach," in 2015 IEEE 8th International Conference on Intelligent Data Acquisition and Advanced Computing Systems: Technology and Applications (IDAACS), 2015, pp. 949-954. https://doi.org/10.1109/IDAACS. 2015.7341444

[8] F. J. Tapia, C. A. Lopez, M. J. Galan, and E. Rubio, "Bayesian Model for Optimization Adaptive e-Learning Process," J. Emerg., vol. 3, no. 1961, pp. 38-52, Feb. 2008.

[9] A. Retbi, F. Merrouch, M. K. Idrissi, and S. Bennani, "Towards a visual Educational Modeling Language for effective learning," Int. J. Comput. Sci., vol. 9, no. 3, pp. 382-390, 2012.

[10] N. M. Seel, T. Lehmann, P. Blumschein, and O. A. Podolskiy, "What is Instructional Design?," in Instructional Design for Learning, Rotterdam: SensePublishers, 2017, pp. 117.

[11] P. Brusilovsky, Adaptive and Intelligent Technologies for Web-based Education, vol. 13, no. 2-4. International AIED Society, 2003.

[12] S. Wan and Z. Niu, "A learner oriented learning recommendation approach based on mixed concept mapping and immune algorithm," Knowledge-Based Syst., vol. 103, pp. 28-40, 2016. https://doi.org/10.1016/j.knosys.2016.03.022

[13] C.-P. Chu, Y.-C. Chang, and C.-C. Tsai, "PC2PSO: personalized e-course composition based on Particle Swarm Optimization," Appl. Intell., vol. 34, no. 1, pp. 141-154, Feb. 2011. https://doi.org/10.1007/s10489-009-0186-7

[14] L. de-Marcos, R. Barchino, J.-J. Martínez, and J.-A. Gutiérrez, "Competency-Based Intelligent Curriculum Sequencing Using Particle Swarms," in 2008 Eighth IEEE International Conference on Advanced Learning Technologies, 2008, pp. 295-297. https://doi.org/10.1109/ICALT.2008.295 
[15] L. de-Marcos, R. Barchino, J.-J. Martinez, and J.-A. Gutierrez, "Competency-Based Intelligent Curriculum Sequencing Using Particle Swarms," Adv. Learn. Technol. 2008. ICALT '08. Eighth IEEE Int. Conf., pp. 295-297, Jul. 2008. https://doi.org/10.1109/ ICALT.2008.295

[16] B. C. L. Christudas, E. Kirubakaran, and P. R. J. Thangaiah, "An evolutionary approach for personalization of content delivery in e-learning systems based on learner behavior forcing compatibility of learning materials," Telemat. Informatics, 2017.

[17] V. Shmelev, M. Karpova, and A. Dukhanov, "An Approach of Learning Path Sequencing Based on Revised Bloom's Taxonomy and Domain Ontologies with the Use of Genetic Algorithms," in Procedia Computer Science, 2015, vol. 66, pp. 711-719. https://doi.org/10.1016/j.procs.2015.11.081

[18] E. Kurilovas, I. Zilinskiene, and V. Dagiene, "Recommending suitable learning scenarios according to learners' preferences: An improved swarm based approach," Comput. Human Behav., vol. 30, pp. 550-557, Jan. 2014. https://doi.org/10.1016/j.chb.2013.06.036

[19] N. Idris, N. Yusof, and P. Saad, "Adaptive Course Sequencing for Personalization of Learning Path Using Neural Network," Int. J. Adv. Soft Comput. Appl, vol. 1, no. 1, 2009.

[20] M. Goyal, A. Choubey, and D. Yadav, "Aggregating evaluation using dynamic weighted intuitionistic fuzzy approach for concept sequencing in an e-learning system," Int. J. Math. Model. Numer. Optim., vol. 7, no. 1, p. 44, 2016. https://doi.org/10.1504/IJMMNO. 2016.074372

[21] M.-J. Huang, H.-S. Huang, and M.-Y. Chen, "Constructing a personalized e-learning system based on genetic algorithm and case-based reasoning approach," Expert Syst. Appl., vol. 33, no. 3, pp. 551-564, Oct. 2007. https://doi.org/10.1016/j.eswa.2006.05.019

[22] S. Miranda, G. R. Mangione, F. Orciuoli, M. Gaeta, and V. Loia, "Automatic generation of assessment objects and Remedial Works for MOOCs," in 2013 12th International Conference on Information Technology Based Higher Education and Training (ITHET), 2013, pp. 1-8. https://doi.org/10.1109/ITHET.2013.6671018

[23] S. Rami, S. Bennani, and M. K. Idrissi, "Towards a method for analyzing learning style using item response theory," in 2017 16th International Conference on Information Technology Based Higher Education and Training (ITHET), 2017, pp. 1-5. https://doi.org/10.1109/ITHET.2017.8067796

[24] C.-M. Chen, C.-Y. Liu, and M.-H. Chang, "Personalized curriculum sequencing utilizing modified item response theory for web-based instruction," Expert Syst. Appl., vol. 30, no. 2, pp. 378-396, Feb. 2006. https://doi.org/10.1016/j.eswa.2005.07.029

[25] Z. W. Zong Woo Geem, J. H. Joong Hoon Kim, and G. V. Loganathan, "A New Heuristic Optimization Algorithm: Harmony Search,” Simulation, vol. 76, no. 2, pp. 60-68, Feb. 2001. https://doi.org/10.1177/003754970107600201

[26] D. Manjarres et al., "A survey on applications of the harmony search algorithm," Eng. Appl. Artif. Intell., vol. 26, no. 8, pp. 1818-1831, Sep. 2013. https://doi.org/10.1016/j.eng appai.2013.05.008

[27] O. Abdel-Raouf, M. Abdel, and -Baset Metwally, "A Survey of Harmony Search Algorithm," Int. J. Comput. Appl., vol. 70, no. 28, pp. 975-8887, 2013.

[28] D. G. Yoo, J. H. Kim, and Z. W. Geem, "Overview of Harmony Search algorithm and its applications in Civil Engineering," Evol. Intell., vol. 7, no. 1, pp. 3-16, Apr. 2014. https://doi.org/10.1007/s12065-013-0100-4 


\section{Authors}

Meriem HNIDA is a Ph.D. Candidate at the Laboratory of Research in Computer Science and Education, Mohammadia School of Engineers, Mohammed V University of Rabat, Morocco. My research project is about the use of educational technology and Competency-based Education to improve Students' Learning. 12 recent publications papers between 2014 and 2017; My interests lie in the fields of Competency modeling and assessment techniques, Ontological engineering, Educational engineering.

Mohammed KHALIDI IDRISSI is a Full Professor at Mohammadia School of Engineers. Doctorate degree in Computer Science in 1986, $\mathrm{PhD}$ in Computer Science in 2009; Former Assistant chief of the Computer Science Department at the Mohammadia School of Engineers (EMI); Pedagogical Tutor of the Computer Science areas at the Mohammadia School of Engineers (EMI) Professor at the Computer Science Department-EMI; 34 recent publications papers between 2014 and 2017; Ongoing research interests: Software Engineering, Information System, Modeling, MDA, ontology, SOA, Web services, eLearning content engineering, tutoring, assessment and tracking.

Samir BENNANI is a Full Professor and Deputy Director of students and academic affairs at Mohammadia School of Engineers. Engineer degree in Computer Science in 1982; PhD in Computer Science in 2005; Professor at the Computer Science Department-EMI; 34 recent publications papers between 2014 and 2017; Ongoing research interests: SI, Modeling in Software Engineering, Information System, eLearning content engineering, tutoring, assessment and tracking.

Article submitted 13 December 2017. Resubmitted 24 March 2018. Final acceptance 24 April 2018. Final version published as submitted by the authors. 